\title{
Comparative Effectiveness of Hypoglycemic Medications Among Veterans
}

\author{
Raya E. Kheirbek, MD, FACP; Farrokh Alemi, PhD; and Manaf Zargoush, MS
}

\begin{abstract}
BACKGROUND: The efficacy of diabetic medications among patients with multiple comorbidities is not tested in randomized clinical studies. It is important to monitor the performance of these medications after marketing approvals.
\end{abstract}

OBJECTIVE: To investigate the risk of all-cause mortality associated with prescription of hypoglycemic agents.

METHODS: We retrospectively examined data from 17,773 type 2 diabetic patients seen from March 2, 1998, to December 13, 2010, in 3 Veterans Administration medical centers. Severity was measured using patients' inpatient and outpatient comorbidities during the last year of visits. Severity-adjusted logistic regression was used to measure the odds ratio for mortality within the study period.

RESULTS: Patients' severity of illness correctly classified mortality for $89.8 \%$ of the patients $(P<0.0001)$. Being younger, married, and white decreased severity adjusted risk of mortality. Exposure to the following medications increased severity adjusted risk of mortality: glyburide (odds ratio $[\mathrm{OR}]=1.804,95 \% \mathrm{Cl}$ from 1.518 to 2.145$)$, glipizide $(\mathrm{OR}=1.566,95 \%$ $\mathrm{Cl}$ from 1.333 to 1.839$)$, rosiglitazone $(\mathrm{OR}=1.805,95 \% \mathrm{Cl}$ from 1.378 to 2.365), chlorpropamide ( $\mathrm{OR}=3.026,95 \% \mathrm{Cl}$ from 1.096 to 8.351 ), insulin $(\mathrm{OR}=2.382,95 \% \mathrm{Cl}$ from 2.112 to 2.686$)$. None of the other medications (metformin, acarbose, glimepiride, pioglitazone, repaglinide, troglitazone, or dipeptidyl peptidase-4) were associated with excess mortality beyond what could be expected from the patients' severity of illness or demographic characteristics. The reported excess mortality could not be explained away by use of other concurrent, nondiabetic classes of medications.

CONCLUSION: Our findings suggest chlorpropamide, glipizide, glyburide, insulin, and rosiglitazone increased severity-adjusted mortality in veterans with type 2 diabetes. A decision aid that could optimize selection of hypoglycemic medications based on patients' comorbidities might increase patients' survival.

J Manag Care Pharm. 2013;19(9):740-44

Copyright $\odot 2013$, Academy of Managed Care Pharmacy. All rights reserved.

\section{What is already known about this subject}

- Rosiglitazone and glyburide may increase cardiac events.

- Metformin and acarbose may have a beneficial effect on all-cause mortality.

- Insulin should be initiated when oral medications are not effective.

\section{What this study adds}

- Rosiglitazone, glyburide, chlorpropamide, glipizide, and insulin were associated with an increase in severity-adjusted all-cause mortality.

- Metformin and acarbose did not have beneficial severity-adjusted impact on all-cause mortality.
$\mathrm{M}$ ore than 171 million people worldwide have type 2 diabetes, a disease associated with at least doubling of the risk of death. ${ }^{1}$ The efficacy of common hypoglycemic medications have been examined in several clinical studies. ${ }^{2-7}$ These studies have intentionally excluded patients with multiple comorbidities in order to focus on the effect of medication on the underlying diabetes. The presence of comorbidities is known to affect adherence to diabetes management protocols. ${ }^{8}$ Investigators have examined the effect of pregnancy, ${ }^{9}$ cancer ${ }^{10}$ depression, ${ }^{11}$ and certain cardiovascular illnesses ${ }^{12}$ on management of diabetic patients. The effect of other comorbidities, or combination of these comorbidities, is not well understood. This study fills the gap in knowledge of how hypoglycemic medications affect all-cause risk-adjusted mortality.

\section{Methods}

This study is a risk-adjusted retrospective analysis of mortality within 1 cohort of patients. The study examined the period between March 2, 1998, and December 12, 2010. A patient's survival during this period was regressed on (a) severity of illness of the patient in the last year of encounters and (b) the extent of use of the medications in the last year of encounters. The findings can be interpreted as severity-adjusted comparative effectiveness of different hypoglycemic medications. In recent years, different methods of studying comparative effectiveness of medications have led to contradictory findings. ${ }^{13}$ Since the focus of interest is on the relationship between mortality and medication taken by a patient, our method of analysis seems reasonable. Our study results would need to be replicated using other study designs, for example, matched case control, before there is confidence in findings.

The project examined data from the electronic health records of diabetic patients seen in the Veterans Administration (VA) Medical Center Capital Area medical centers, including those in Baltimore, Maryland; Martinsburg, West Virginia; Perry Point, Maryland; and Washington, DC. The analysis was done on 19,223 diabetic patients, 17,773 of whom met the eligibility criteria.

\section{Eligibility Criteria}

The eligibility criteria were as follows:

Diabetic: The patient must have had 2 diagnoses of diabetes (International Classification of Diseases, Ninth Revision, Clinical 
Comparative Effectiveness of Hypoglycemic Medications Among Veterans

TABLE 1 Logistic Regression of Mortality Associated with Exposure to Hypoglycemic Medications

\begin{tabular}{|c|c|c|c|c|c|c|c|c|c|}
\hline & Number & & & Wald & & & & $95 \% \mathrm{CI}$ & $\operatorname{Exp}(B)$ \\
\hline & Exposed & B & SE & Statistic & $\mathrm{df}$ & Sig & OR & Lower & Upper \\
\hline Metformin & 5,629 & -0.125 & 0.076 & 2.736 & 1 & 0.098 & 0.883 & 0.761 & 1.023 \\
\hline Glyburide & 1,732 & 0.59 & 0.088 & 44.706 & 1 & 0 & 1.804 & 1.518 & 2.145 \\
\hline Glipizide $^{\mathrm{a}}$ & 2,572 & 0.448 & 0.082 & 29.748 & 1 & 0 & 1.566 & 1.333 & 1.839 \\
\hline Rosiglitazone $^{a}$ & 463 & 0.591 & 0.138 & 18.408 & 1 & 0 & 1.805 & 1.378 & 2.365 \\
\hline Acarbose & 96 & 0.369 & 0.294 & 1.579 & 1 & 0.209 & 1.446 & 0.813 & 2.57 \\
\hline Chlorpropamide & 23 & 1.107 & 0.518 & 4.569 & 1 & 0.033 & 3.026 & 1.096 & 8.351 \\
\hline Glimepiride & 149 & 0.194 & 0.299 & 0.421 & 1 & 0.516 & 1.214 & 0.676 & 2.179 \\
\hline Pioglitazone & 102 & 0.416 & 0.327 & 1.62 & 1 & 0.203 & 1.516 & 0.799 & 2.878 \\
\hline Repaglinide & 30 & -0.406 & 0.604 & 0.453 & 1 & 0.501 & 0.666 & 0.204 & 2.174 \\
\hline Insulin ${ }^{\mathrm{a}}$ & 5,236 & 0.868 & 0.061 & 200.722 & 1 & 0 & 2.382 & 2.112 & 2.686 \\
\hline $\mathrm{DPP} 4$ & 24 & 0.294 & 0.793 & 0.137 & 1 & 0.711 & 1.342 & 0.284 & 6.344 \\
\hline Severity of illness ${ }^{\mathrm{a}}$ & 17,773 & 13.89 & 0.326 & $1,819.207$ & 1 & 0 & $1,077,039$ & $568,901.1$ & $2,039,043$ \\
\hline Birth yeara & 17,773 & -0.031 & 0.003 & 147.228 & 1 & 0 & 0.969 & 0.964 & 0.974 \\
\hline Male & 16,924 & 0.008 & 0.183 & 0.002 & 1 & 0.965 & 1.008 & 0.705 & 1.442 \\
\hline White ${ }^{a}$ & 4,638 & -0.4 & 0.067 & 35.427 & 1 & 0 & 0.67 & 0.587 & 0.765 \\
\hline Hispanic & 5,321 & -0.082 & 0.063 & 1.702 & 1 & 0.192 & 0.921 & 0.815 & 1.042 \\
\hline Married $^{a}$ & 8,164 & -0.206 & 0.058 & 12.658 & 1 & 0 & 0.814 & 0.726 & 0.912 \\
\hline Constant $^{\mathrm{a}}$ & & 55.551 & 5.029 & 122.033 & 1 & 0 & $1.34 \mathrm{E}+24$ & & \\
\hline
\end{tabular}

Modification [ICD-9-CM] codes 250.xx, 357.2, 362.0x, 366.41, or 648.0x, where $\mathrm{x}$ is any digit).

Minimum data required: Patients with less than 180 days between first and last visits were excluded on suspicion that these patients had too few encounters or may have received care elsewhere.

Inconsistent date of death: Patients with a recorded date of death 30 days prior to their last visit were not included in the analysis. A 30-day interval was allowed because occasionally test results become available after date of death, or visits are coded to have been completed after date of death.

\section{Dependent Variable}

The primary end-point variable in this study was all-cause mortality during the study time period. Health system data do not usually reflect death at home or outside the health system. The VA systematically checks with Social Security records to update its files on deaths reported within other systems.

\section{Independent Variables}

The independent variables were as follows:

Severity of illness: The composite for severity of illness was calculated from patients' diagnoses during the last year of patients' visits. We used a variant of the Alemi and Walter's severity index, a patented algorithm that assigns severity scores to combinations of diagnoses/procedure codes. ${ }^{14}$ Appendix A (available in online article) shows how this measure of severity was constructed; a brief description is provided here:

- The average mortality associated with each diagnosis and each pair of diagnoses was calculated. One unit of increase in this score is equivalent to a 1-point increase in probability of mortality.

- Pairs of diagnoses that did not repeat at least 5 times across patients were excluded from the analysis.

- A patient's overall severity for each encounter was scored as the average of all of the patient's pairs of diagnoses for hospitalization encounters and single diagnoses for outpatient visits.

The severity scores for each individual diagnosis could theoretically range from 0 to 1 , and they actually ranged from .001 to 0.803 . The mean severity score was 0.167 . The median was 0.140 , and the standard deviation was 0.091 . The more severe the patients' diagnoses during hospital or outpatient encounters, the higher the average severity score for each patient.

Current medications: This set of independent variables measured exposure to various hypoglycemic medications (metformin, glyburide, glipizide, rosiglitazone, acarbose, chlorpropamide, glimepiride, pioglitazone, tolazamide, repaglinide, troglitazone, insulin, and dipeptidyl peptidase-4 [DPP4]) and 76 classes of nondiabetic medications (see Appendix B, available in online article). The number of patients who were exposed to each of the hypoglycemic medications is reported in Table 1. The definition of "exposure to medication" was set to any use of the medication independent of dose or days of use. This was done in order to have consistent definitions across classes of medications, where use of different medications within the same class prohibited calculation of cumulative dosage prescribed. Nevertheless, the definition of "exposure" introduced 


\section{FIGURE 1 Relationship Between Composite Severity and Mortality}

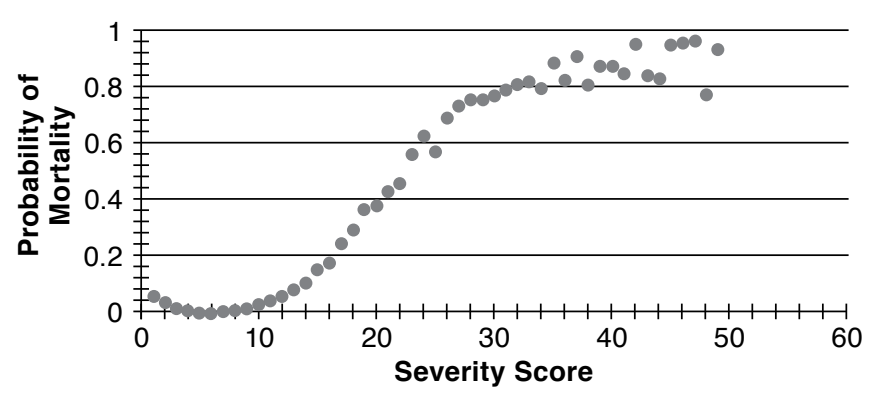

possible errors in the data. To examine the extent of these errors, we repeated our analysis, replacing "exposure to hypoglycemic medications" with the "sum of daily dose prescribed." The findings of the study did not change; the same medications were found to be associated with increased severity-adjusted mortality. Another problem with our measure of exposure to medications is that it does not reflect actual medication taken but medications prescribed. It is well known that patients do not take medications as prescribed or purchased. ${ }^{15}$ We did not have access to data on medication adherence.

Demographics: Year of birth, gender, race, marital status, and ethnicity were used to adjust for differences in mortality. Missing demographic variables were assumed to be the mode of the data. The distribution of the demographic variables is provided in the first column of Table 1 .

\section{Statistical Analysis}

Data were analyzed using Logistic Regression. The statistical package used was PASW Statistics 18, release version 18.0.0 (SPSS, Inc., 2009, Chicago, IL, www.spss.com). Patients' mortality was regressed on the composite severity measure, demographic measures, concurrent nondiabetic medications, and a set of variables measuring exposure to diabetes medications. This approach controlled for severity of diabetic illness before attributing observed mortality to specific medications.

\section{Results}

A key construct, on which much of the remaining analysis rests, was our measure of composite for severity of illness. Figure 1 illustrates the relationship between this index and the observed rates of mortality. To construct this figure, we divided severity scores into uniform intervals and calculated the mortality rate for each interval. On the x-axis are the severity scores, and on the $y$-axis is the average mortality for patients who received the score. Each point on the graph represented a different number of patients (minimum of 15 and a maximum of 1,316 patients), as some severity intervals were more common. The graph fits our expectations of the relationship between severity of illness and mortality rates. Low severity scores were associated with low mortality rates, and high scores were associated with high mortality rates. For scores between 20 and 30, there was a rapid increase in mortality rate proportional to the severity score. At the very low and the very high scores, there were occasional errors in the severity score, where an increase in severity was not associated with an increase in mortality. Nevertheless, as a whole, the composite severity score was highly predictive of mortality. It correctly classified $89.6 \%$ of observed mortality (chi-square $=142.95, P<0.0001$ ).

Table 1 provides the odds ratio (OR) associated with different hypoglycemic medications and severity-adjusted mortality. An OR larger than 1 indicates medications that increased mortality. An OR less than 1 indicates reduced mortality rate. The regression equation correctly predicted $89.8 \%$ of patients examined. The severity index was the best predictor of mortality. Married, younger, and white patients had a lower risk of mortality. After adjustments were made for severity of illness and patient demographics, the remaining variance in mortality was explained by exposure to 5 medications, listed in order of impact on risk-adjusted mortality: glipizide $(\mathrm{OR}=1.566)$, glyburide $(\mathrm{OR}=1.804)$, rosiglitazone $(\mathrm{OR}=1.805)$, insulin $(\mathrm{OR}=2.382)$, and chlorpropamide $(\mathrm{OR}=3.026)$. None of the other medications (metformin, acarbose, glimepiride, pioglitazone, tolazamide, repaglinide, troglitazone, and DPP4) were associated with excess mortality beyond what could be expected from the patients' severity of illness or demographic characteristics.

The data we had were observational data. Therefore, it is not clear that the reported mortality is related to the hypoglycemic medication or a common medication that is prescribed with it. To partially control for possible interactions, we repeated the analysis and included 76 common classes of concurrent medications (Appendix B, available in online article). Insulin, glyburide, glipizide, and rosiglitazone continued to be associated with statistically significant increased mortality. This analysis further confirmed that classes of nondiabetic medications do not explain away the mortality we have attributed to diabetic medications.

\section{Discussion}

We presented data on the association between hypoglycemic medications and all-cause severity-adjusted mortality.

This analysis was done on a relatively large sample of diabetic patients. In large samples, even small differences in outcome could be statistically significant. The data were adjusted for patients' severity of illness by examining all patient diagnoses and scoring each pair of diagnoses separately based on the observed mortality within the pair. Analysis of the measure of severity indicated that it performed as expected and that it explained a large percentage of variation in mortality. These data increase confidence that the measure had adequately captured patients' severity of illness. 
One finding of our study was the association of insulin with higher severity-adjusted mortality (see Table 1). Traditionally, insulin has been tried after diabetic patients have not been responsive to oral medications. Recent data suggest that earlier use of insulin may be beneficial; replacing oral medications with insulin at onset of treatment of diabetes may lead to better control of hemoglobin Alc and reduced complications. ${ }^{16}$ However, despite better glycemic control, all-cause mortality (from hypoglycemia, weight gain, and colorectal cancer) may increase. ${ }^{17}$ Our data, while not directly addressing the timing of start of insulin, suggest that exposure to insulin is associated with higher all-cause mortality, even after adjusting for severity of illness. These data fit into the clinical perspective that insulin should be delayed until oral medications fail to maintain glycemic control.

Another finding of our study was that rosiglitazone was associated with increased severity-adjusted all-cause mortality (see Table 1). The original approval of rosiglitazone was based on the ability of the drug to reduce blood glucose and glycated hemoglobin levels. ${ }^{18}$ One potential contributing factor may be the adverse effect of the drug on serum lipids. A 2007 study found rosiglitazone was associated with a significant increase in the risk of myocardial infarction and with an increase in the risk of death from cardiovascular causes that had borderline significance. ${ }^{19}$ The Diabetes Outcome Progression Trial demonstrated 5-year failure rates with rosiglitazone to be lower than metformin or glyburide. Rosiglitazone was associated with more weight gain and edema than either metformin or glyburide but with fewer gastrointestinal events than metformin and with less hypoglycemia than glyburide. ${ }^{20}$ The current study complements these findings by providing additional data on the impact of rosiglitazone on all-cause mortality.

The published literature suggests that metformin should have reduced severity-adjusted mortality. ${ }^{21}$ Our study did not find a statistically significant relationship between metformin and severity-adjusted mortality. Before severity adjustment, there was a beneficial effect $(\mathrm{OR}=0.320,95 \%$ confidence interval [CI] from 0.283 to 0.362 ). After severity adjustment, there was not $(\mathrm{OR}=0.883$, CI from 0.761 to 1.023$)$. This suggests that the beneficial effects attributed to metformin might be due to use of this medication by patients who are younger and less severely ill. Once severity of illness and demographics are taken into account, metformin had no statistically significant impact on mortality (see Table 1).

Post-FDA-release surveillance studies have shown that acarbose may have a beneficial effect on mortality.22 Our data contradict this finding. Prior to severity adjustment, a detrimental effect was associated with acarbose (OR $=2.141,95 \% \mathrm{CI}$ from 1.335 to 3.435 ). After severity and demographic adjustment, acarbose had neither detrimental nor beneficial impact on mortality (see Table 1). The study that had shown beneficial effects for acarbose was carried out on Chinese patients. The differences between our study population and Chinese patients may explain the contradictory findings. Our study was limited to U.S. veterans. The set of comorbidities may be different in different populations.

\section{Limitations}

This study was limited because it was an observational study. In observational studies, a number of factors may cause the outcome. Some of these factors are controlled for, but it is not possible to statistically control all possible causes. This study statistically controlled for the patients' severity of illness, concurrent medication classes, and patient demographics. We attributed mortality not explained by these factors to the patients' diabetes medications. Such attributions may be in error. There are other causes of mortality (e.g., obesity, poor diet, substance abuse, lack of exercise) for which we did not statistically control.

\section{Conclusion}

These data show that glyburide, glipizide, rosiglitazone, chlorpropamide, and insulin led to severity-adjusted higher mortality rates. It is important to explore under what conditions and for which patients these medications may not be beneficial. Given the large number of hypoglycemic medications and comorbid conditions, it may be important to develop tools that can help selection of optimal medications for diabetic patients that present with comorbidities.

\section{Authors}

RAYA E. KHEIRBEK, MD, FACP, is Deputy Chief of Staff, Veterans Affairs Medical Center, The George Washington University School of Medicine and Health Sciences, Washington, DC; FARROKH ALEMI, $\mathrm{PhD}$, is Chief of Performance Improvement, Veterans Affairs Medical Center, Washington, DC, and Professor, University of South Florida Department of Health Policy and Management, Tampa, Florida; and MANAF ZARGOUSH, MS, is a PhD Candidate in Decision Sciences, ESSEC Business School and Healthcare Operations Management, McGill University, Montreal, Quebec, Canada.

AUTHOR CORRESPONDENCE: Raya E. Kheirbek, MD, FACP, District of Columbia Veterans Affairs Medical Center, 50 Irving St., NW, Washington, DC 20422. E-mail: raya.kheirbek@va.gov.

\section{DISCLOSURES}

Financial support for this study was provided entirely by appropriation from Veterans Affairs Office of System Change. The funding agreement ensured the authors' independence in designing the study, interpreting the data, writing, and publishing the report. Kheirbek and Alemi are employed by the sponsor

Study concept and designed were contributed by Kheirbek and Alemi. Data collection was performed by Alemi and Kheirbek, and data analysis was done by Alemi and Zargoush. Data were interpreted by Kheirbek, Alemi, and Zargoush, who also wrote and revised the manuscript. 


\section{REFERENCES}

1. Wild S, Roglic G, Green A, Sicree R, King H. Global prevalence of diabetes: estimates for the year 2000 and projections for 2030. Diabetes Care. 2004;27(5):1047-53

2. DREAM Trial Investigators, Dagenais GR, Gerstein HC, et al. Effects of ramipril and rosiglitazone on cardiovascular and renal outcomes in people with impaired glucose tolerance or impaired fasting glucose: results of the Diabetes REduction Assessment with ramipril and rosiglitazone Medication (DREAM) trial. Diabetes Care. 2008;31(5):1007-14.

3. Gerstein HC, Riddle MC, Kendall DM, et al. Glycemia treatment strategies in the Action to Control Cardiovascular Risk in Diabetes (ACCORD) trial. Am J Cardiol. 2007;99(12A):34i-43i.

4. ADVANCE Collaborative Group, Patel A, MacMahon S, et al. Intensive blood glucose control and vascular outcomes in patients with type 2 diabetes. N Engl J Med. 2008;358(24):2560-72.

5. Kageyama S, Ueda S, Mochizuki K, et al. Optimal Combination of Effective ANtihypertensives (OCEAN) study: a prospective, randomized, open-label, blinded endpoint trial-rationale, design and results of a pilot study in Japan. Hypertens Res. 2012;35(2):221-27.

6. Grant RW, Devita NG, Singer DE, Meigs JB. Polypharmacy and medication adherence in patients with type 2 diabetes. Diabetes Care. 2003;26(5):1408-12

7. Wright EE Jr, Stonehouse AH, Cuddihy RM. In support of an early polypharmacy approach to the treatment of type 2 diabetes. Diabetes Obes Metab. 2010;12(11):929-40.

8. El-Kebbi IM, Ziemer DC, Gallina DL, et al. Diabetes in urban AfricanAmericans. XV. Identification of barriers to provider adherence to management protocols. Diabetes Care. 1999;22(10):1617-20

9. Kitzmiller JL, Block JM, Brown FM, et al. Managing preexisting diabetes for pregnancy: summary of evidence and consensus recommendations for care. Diabetes Care. 2008;31(5):1060-79.

10. Tsugane S, Inoue M. Insulin resistance and cancer: epidemiological evidence. Cancer Sci. 2010;101(5):1073-79.
11. Kivimäki M, Hamer M, Batty GD, et al. Antidepressant medication use, weight gain, and risk of type 2 diabetes: a population-based study. Diabetes Care. 2010;33(12):2611-16

12. Stolar MW, Hoogwerf BJ, Gorshow SM, Boyle PJ, Wales DO. Managing type 2 diabetes: going beyond glycemic control. J Manag Care Pharm. 2008;14(5 Suppl B):S2-S19. Available at: http://www.amcp.org/WorkArea/ DownloadAsset.aspx?id=13819.

13. Lohr KN. Comparative effectiveness research methods: symposium overview and summary. Med Care. 2010;48(6 Suppl):S3-S6.

14. Alemi F, Walters SR. A mathematical theory for identifying and measuring severity of episodes of care. Qual Manag Health Care. 2006;15(2):72-82. 15. Greevy RA Jr, Huizinga MM, Roumie CL, et al. Comparisons of persistence and durability among three oral antidiabetic therapies using electronic prescription-fill data: the impact of adherence requirements and stockpiling. Clin Pharmacol Ther. 2011;90(6):813-19.

16. Simons WR, Vinod HD, Gerber RA, Bolinder B. Does rapid transition to insulin therapy in subjects with newly diagnosed type 2 diabetes mellitus benefit glycaemic control and diabetes-related complications? A German population-based study. Exp Clin Endocrinol Diabetes. 2006;114(9):520-26. 17. Chiasson JL. Early insulin use in type 2 diabetes: what are the cons? Diabetes Care. 2009;32(Suppl 2):S270-S74.

18. Pouwels KB, van Grootheest K. The rosiglitazone decision process at FDA and EMA. What should we learn? Int J Risk Saf Med. 2012;24(2):73-80 19. Nissen SE, Wolski K. Effect of rosiglitazone on the risk of myocardial infarction and death from cardiovascular causes. N Engl J Med. 2007;356(24):2457-71.

20. Kahn SE, Haffner SM, Heise M, et al. Glycemic durability of rosiglitazone, metformin, or glyburide monotherapy. N Engl J Med. 2006; 355(23):2427-43.

21. Jabbour S, Ziring B. Advantages of extended-release metformin in patients with type 2 diabetes mellitus. Postgrad Med. 2011;123(1):15-23.

22. Pan CY, Landen H. Post-marketing surveillance of acarbose treatment in patients with type 2 diabetes mellitus and subjects with impaired glucose tolerance in China. Clin Drug Investig. 2007;27(6):397-405. 


\section{APPENDIX A Method of Scoring Composite Severity Index}

The composite severity was scored based on pairs of diagnoses. First, the average mortality associated with each pair of diagnoses that repeated at least 5 times was calculated:

Where

$$
p_{j, k}=\frac{\sum_{i, e} D_{i, e, j, k} / n_{i}}{D_{\max }}
$$

$p_{j, k} \quad$ was the estimated probability of mortality in pair of diagnoses "j" and "k," where " $\mathrm{j}$ " is the primary diagnosis, if this information is available, otherwise " $\mathrm{j}$ " is the first listed diagnosis.

$D_{\max }$ was the maximum number of days till mortality, for any pair of diagnoses in any encounter of any patient that died.

$n_{i} \quad$ was the number of diagnoses pairs for patient "i" across all encounters.

$D_{i} \quad$ was the number of days between mortality of patient " $i$ " and diagnoses pair "j" and "k" in encounter "e," of patient "i." For patients who did not die, this was set to $D_{\max }$.

Then, the overall severity for an encounter "e" of patient " $i$ " was calculated as the average of the severity of diagnoses within that encounter:

Where

$p_{j, k} \quad$ was the estimated probability of mortality in pair of diagnoses " $\mathrm{j}$ " and "k" within encounter "e."

$r_{i} \quad$ was the number of unique pairs of diagnoses within encounter "e."

Finally, the overall severity for patient "i" was calculated as:

Where

$$
O_{i}=\frac{\sum_{e} O_{i, e}}{m_{i}}
$$

$O_{i} \quad$ was the overall severity of illness of patient "i."

$O_{i, e} \quad$ was the severity of encounter "e" for patient "i."

$m_{i} \quad$ was the number of encounters for patient "i."

The severity index included diagnoses within the following components: (1) health status diagnoses; (2) endocrine nutrition and metabolic diseases; (3) circulatory diseases; (4) mental diseases and disorders; (5) musculoskeletal and connective tissue; (6) substance use and disorders; (7) eye problems, (8) nervous system; (9) ear, nose, mouth and throat problems; (10) kidney and urinary tract problems; (11) respiratory system problems; (12) digestive system; (13) skin, breast, and subcutaneous tissue problems; (14) male reproductive system problems; (15) hepatobiliary and pancreas diseases; (16) diseases of myeloproliferative neoplasia; (17) injury, poisoning, and drug toxicity; (18) HIV infection, and (19) infectious and parasitic diseases. Each diagnosis was scored independent of its classification in a particular component, and these disease categories are presented as a general guide to the index and not as classes of diagnoses used for scoring. Table Al shows the top 5 most serious diagnoses within each category.

\begin{tabular}{|c|c|}
\hline Component & \\
\hline Diabetes type & $\begin{array}{l}\text { (250.72) Diabetes mellitus with peripheral circulatory disorders type II or unspecified type uncontrolled, (250.12) Diabetes mel- } \\
\text { litus with ketoacidosis type II or unspecified type uncontrolled, (250.91) Diabetes mellitus with unspecified complication type I } \\
\text { not stated as uncontrolled, (250.62) Diabetes mellitus with neurological manifestations type II or unspecified type uncontrolled, } \\
\text { (250.71) Diabetes mellitus with peripheral circulatory disorders type I not stated as uncontrolled }\end{array}$ \\
\hline Health status & $\begin{array}{l}\text { (V64.2) Surgical or other procedure not carried out because of patients decision, (V44.1) Gastrostomy status, (V66.7) Encounter } \\
\text { for palliative care, (780.94) Early satiety, (780.94) Other general symptoms }\end{array}$ \\
\hline $\begin{array}{l}\text { Endocrine nutrition and } \\
\text { metabolic diseases }\end{array}$ & $\begin{array}{l}\text { (783.41) Failure to thrive, (269.8) Other nutritional deficiency, (783.7) Adult failure to thrive, (783.4) Lack of expected normal } \\
\text { physiological development, (255.4) Carticoadrenal insufficiency }\end{array}$ \\
\hline Circulatory diseases & $\begin{array}{l}\text { (427.5) Cardiac arrest, (428.22) Chronic systolic heart failure, (250.72) Diabetes with peripherial circulatory disorders, Type II or } \\
\text { unspecified type, uncontrolled, (443.89) Other peripheral vascular disease, (798.1) Instantaneous death }\end{array}$ \\
\hline $\begin{array}{l}\text { Mental diseases and } \\
\text { disorders }\end{array}$ & $\begin{array}{l}\text { (310.0) Frontal lobe syndrome, (290.20) Senile dementia with delusional features, (290.10) Pre-senile dementia, uncomplicated, } \\
\text { (301.81) Narcissistic personality disorders, (300.09) Other anxiety disorders }\end{array}$ \\
\hline $\begin{array}{l}\text { Musculoskeletal and } \\
\text { connective tissue }\end{array}$ & $\begin{array}{l}\text { (198.5) Secondary malignant neoplasm of bone and bone marrow, (737.10) Kyphosis, acquired postural, (725.) Polymyalgia rheu- } \\
\text { matic, (730.17) Chronic osteomyelitis involving ankle and foot, (446.5) Giant cell artheritisco }\end{array}$ \\
\hline $\begin{array}{l}\text { Substance use and } \\
\text { disorders }\end{array}$ & $\begin{array}{l}\text { (291.2) Alcohol-induced persisting dementia, (305.03) Alcohol abuse in remission, (304.03) Opioid-type dependence in remission, } \\
\text { (304.00) Opioid-type dependence unspecified, (304.71) Combinations of opioid-type drug with any other drug dependence, } \\
\text { continuous use }\end{array}$ \\
\hline
\end{tabular}

\section{TABLE A1 Serious Diagnoses Among Diabetic Patients}




\section{TABLE A1 Serious Diagnoses Among Diabetic Patients (continued)}

\begin{tabular}{|c|c|}
\hline ent & \\
\hline te problems & $\begin{array}{l}\text { (365.63) Glaucoma associated with vascular disorders, (365.60) Glaucoma associated with unspecified ocular disorder, (190.6) } \\
\text { Malignant neoplasm of choroid, (376.30) Exophthalmos, unspecified, (371.57) Endothelial corneal dystrophy }\end{array}$ \\
\hline Nervous system & $\begin{array}{l}\text { (191.8) Malignant neoplasm of other parts of brain, (198.3) Secondary malignant neoplasm of brain and spinal cord, (742.59) } \\
\text { Other specified congenital anomalies of spinal cord, (432.1) Subdural hemorrhage, (952.2) Lumbar spinal cord injury without spi- } \\
\text { nal bone injury }\end{array}$ \\
\hline $\begin{array}{l}\text { Ear, nose, mouth, and } \\
\text { throat problems }\end{array}$ & $\begin{array}{l}\text { (389.8) Other specified forms of hearing loss, (142.0) Malignant neoplasm of parotid gland, (195.0) Malignant neoplasm of head, } \\
\text { face, and neck, (161.0) Malignant neoplasm of glottis, (382.00) Acute suppurative otitis media without spontaneous rupture of } \\
\text { eardrum }\end{array}$ \\
\hline $\begin{array}{l}\text { Kidney and urinary } \\
\text { tract problems }\end{array}$ & $\begin{array}{l}\text { (583.2) Nephritis and nerphopathy, not specified as acute or chronic, with lesion of membranoproliferative glomerulonephritis, } \\
\text { (188.8) Malignant neoplasm of other specified sites of bladder, (189.0) Malignant neoplasm of kidney, except pelvis, (188.1) } \\
\text { Malignant neoplasm of dome of urinary bladder, (593.4) Other ureteric obstruction }\end{array}$ \\
\hline $\begin{array}{l}\text { Respiratory system } \\
\text { oroblems }\end{array}$ & $\begin{array}{l}\text { (197.0) Secondary malignant neoplasm of lung, (512.1) Iatrogenic pneumothorax, (162.4) Malignant neoplasm of middle lobe, } \\
\text { bronchus or lung, (162.9) Malignant neoplasm of bronchus and lung, unspecified, (231.2) Carcinoma in situ of bronchus and lung }\end{array}$ \\
\hline Digestive system & $\begin{array}{l}\text { (150.5) Malignant neoplasm of lower third of esophagus, (569.81) Fistula of intestine, excluding rectum and anus, (537.3) Other } \\
\text { obstruction of duodenum, (008.46) Intestinal infection due to other anaerobes, (787.6) Incontinence of feces }\end{array}$ \\
\hline $\begin{array}{l}\text { Male reproductive } \\
\text { system problems }\end{array}$ & $\begin{array}{l}\text { (185) Malignant neoplasm of prostate, (112.2) Candidiasis of other urogenital sites, (607.81) Blanitis xerotica obliterans, (600) } \\
\text { Hyperplasia of prostate, (607.84) Impotence of organic origin }\end{array}$ \\
\hline $\begin{array}{l}\text { Hepatobiliary and } \\
\text { pancreas diseases }\end{array}$ & $\begin{array}{l}\text { (157.0) Malignant neoplasm of head of pancreas, (157.9) Malignant neoplasm of pancreas, part unspecified, (571.6) Biliary cirrho- } \\
\text { sis, (155.0) Malignant neoplasm of liver, primary, (155.2) Malignant neoplasm of liver, not specified as primary or secondary }\end{array}$ \\
\hline $\begin{array}{l}\text { Diseases of myeloprolif- } \\
\text { erative neoplasia }\end{array}$ & $\begin{array}{l}\text { (201.42) Hodgkin's disease, lymphocytic predominance involving intrathoracic lymph nodes, (208.00) Acute leukemia of unspeci- } \\
\text { fied cell type without remission, (238.6) Neoplasm of uncertain behavior of plasma cells, (199.0) Disseminated malignant neo- } \\
\text { plasm, (273.8) Other disorders of plasma protein metabolism }\end{array}$ \\
\hline $\begin{array}{l}\text { Injury, poisoning, and } \\
\text { drug toxicity }\end{array}$ & $\begin{array}{l}\text { (886.1) Traumatic amputation of other fingers, complete, partial, complicated, (897.2) Traumatic amputation of legs, complete, } \\
\text { partial, unilateral, at or above knee, without mention of complication, (897.6) Traumatic amputation of legs, complete, partial, } \\
\text { bilateral, any level, without mention of complication, (897.0) Traumatic amputation of legs, complete, partial, unilateral, below } \\
\text { knee, without mention of complication, (964.2) Poisoning by anticoagulants }\end{array}$ \\
\hline $\begin{array}{l}\text { Human immunodefi- } \\
\text { ciency virus infection }\end{array}$ & $\begin{array}{l}\text { (042) Human immunodeficiency virus, HIV, disease, (478.19) Other disease of nasal cavity and sinuses, (780.96) Generalized } \\
\text { pain, (525.61) Open restoration margins, (523.42) Chronic periodontitis, generalized }\end{array}$ \\
\hline $\begin{array}{l}\text { Infectious and parasitic } \\
\text { diseases }\end{array}$ & $\begin{array}{l}\text { (041.89) Bacterial infection die to other specified bacteria, (097.0) Late syphilis, unspecified, (097.1) Latent syphilis, unspecified, } \\
\text { (038.9) Unspecified septicemia, (136.9) Unspecified infectious and parasitic diseases }\end{array}$ \\
\hline d subcu- & nention of complication, (707.07) Decubitus ulcer, heel, (039.0) \\
\hline
\end{tabular}




\section{APPENDIX B Seventy-Six Nondiabetic Medications as Independent Variables}

This study reports the association between diabetic medications and excess mortality. It is possible that this association might be due to concurrent nondiabetic medications. This appendix lists nondiabetic medications and supplies that were used as an alternative explanation for excess mortality. The analysis was done by the entire class, not by items within the class. The following medication classes were included:

antidotes/deterrents, other

antihistamines, ethanolamine

antihistamines, piperazine

antihistamines, butyrophenone

antihistamines, other

(inactive) penicillin g-related

penicillins

penicillins, amino derivatives

erythromycins/macrolides

antivirals

anti-infectives, other

parasympatholytics

anticoagulants

platelet aggregation inhibitors

blood formation products

benzodiazepine derivative sedatives/ hypnotics

sedatives/hypnotics, other

anticonvulsants

tricyclic antidepressants

central nervous system medications, other digitalis glycosides

alpha blockers/related calcium

channel blockers

antianginals

antihypertensive combinations

antihypertensives, other

thiazides/related diuretics

loop diuretics

potassium sparing/combinations diuretics

angiotensin II inhibitor

antibacterial, topical

antifungal, topical

anti-inflammatory, topical

soaps/shampoos/soap-free cleansers

analgesics, topical

dermatologicals, topical other

bulk-forming laxatives

hyperosmotic laxatives

stimulant laxatives

stool softener histamine antagonists

gastric medications, other

herbs/alternative therapies

glucocorticoids

thyroid supplements

hormones/synthetics/modifiers,

other

immune suppressants

investigational agents

skeletal muscle relaxants

antigout agents

anti-inflammatories, nasal

nasal and throat, topical, other

beta-blockers, topical ophthalmic

antiglaucoma combinations, topical ophthalmic

antiglaucoma, other

anti-inflammatories, topical

ophthalmic

eye washes/lubricants

anti-inflammatories, inhalation

bronchodilators, sympathomimetic, inhalation bronchodilators, anticholinergic

antiasthma, other

non-opioid-containing antitussives/ expectorants

enteral nutrition

iron

calcium

potassium

cyanocobalamin

folic acid/leucovorin

nicotinic acid

multivitamins

multivitamins with minerals

pads, gauze, sterile

pads, gauze with medication added

bandages/dressings, other

diapers

syringes, insulin, injection

needles, injection

supplies, other 